\title{
Special relativity induced by granular space
}

\author{
Petr Jizba $^{1,2, a}$, Fabio Scardigli ${ }^{3,4, b}$ \\ ${ }^{1}$ FNSPE, Czech Technical University in Prague, Břehová 7, 11519 Prague 1, Czech Republic \\ ${ }^{2}$ ITP, Freie Universität Berlin, Arnimallee 14, 14195 Berlin, Germany \\ ${ }^{3}$ Yukawa Institute for Theoretical Physics, Kyoto University, Kyoto 606-8502, Japan \\ ${ }^{4}$ Dipartimento di Matematica, Politecnico di Milano, Piazza Leonardo da Vinci 32, 20133 Milan, Italy
}

Received: 20 May 2013 / Published online: 6 July 2013

(C) The Author(s) 2013. This article is published with open access at Springerlink.com

\begin{abstract}
We show that the special relativistic dynamics, when combined with quantum mechanics and the concept of superstatistics, can be interpreted as arising from two interlocked non-relativistic stochastic processes that operate at different energy scales. This framework leads to Feynman amplitudes that are, in the Euclidean regime, identical to the transition probability of a Brownian particle propagating through a granular space. For illustration we consider the dynamics and the propagator of a Klein-Gordon particle. Implications for deformed special relativity, quantum field theory, quantum gravity and cosmology are also discussed.
\end{abstract}

\section{Introduction}

The concept of "emergence" plays an important role in quantum field theory (QFT) and, in particular, in condensed matter and particle physics, since it embodies the essential feature of systems with several interlocked time scales. In these systems the observed macroscopic-scale dynamics and related degrees of freedom differ drastically from the actual underlying microscopic-scale physics [1-3]. Superstatistics provides a specific realization of this paradigm: It predicts that the emergent behavior can be often regarded as a superposition of several statistical systems that operate at different spatio-temporal scales [4-7]. In particular, many applications have recently been reported, in hydrodynamic turbulence [8], turbulence in quantum liquids [9], pattern forming systems [10], high-energy physics [11, 12], etc.

The essential assumption of the superstatistics scenario is the existence of sufficient spatio-temporal scale separations between relevant dynamics within the studied system,

\footnotetext{
a e-mail: p.jizba@fjif.cvut.cz

be-mail: fabio@phys.ntu.edu.tw
}

so that the system has enough time to relax to a local equilibrium state and to stay within it for some time. In practical applications one is typically concerned with two scales. Following Refs. [4-6], we consider an intensive parameter $\zeta$ that fluctuates on a much larger time scale than the typical relaxation time of the local dynamics. The random variable $\zeta$ can be in practice identified, e.g., with the inverse temperature [4-7], friction constant [13], volatility [14] or einbein [15]. On an intuitive ground, one may understand the superstatistics by using the adiabatic Ansatz. Namely, the system under consideration, during its evolution, travels within its state space $X$ (described by a state variable $A \in X$ ) which is partitioned into small cells characterized by a sharp value of some intensive parameter $\zeta$. Within each cell, the system is described by the conditional distribution $p(A \mid \zeta)$. As $\zeta$ varies adiabatically from cell to cell, the joint distribution of finding the system with a sharp value of $\zeta$ in the state $A$ is $p(A, \zeta)=p(A \mid \zeta) p(\zeta)$ (Bayes theorem). The resulting macro-scale (emergent) statistics $p(A)$ for finding system in the state $A$ is obtain by eliminating the nuisance parameter $\zeta$ through marginalization, that is

$p(A)=\int p(A \mid \zeta) p(\zeta) d \zeta$

Due to a sufficient time-scale separation between relevant dynamics superstatistic qualifies as a form of slow modulation [16].

In this Letter, we cast the Feynman transition amplitude of a relativistic particle into a form which (after being analytically continued to imaginary times) coincides with a superstatistics marginal probability (1). The derivation is based on the Lévy-Khinchine theorem for infinitely divisible distributions [17, 18], and for illustration we consider the dynamics and the propagator of a Klein-Gordon (i.e., neutral spin-0) particle. Our reasonings can be also extended to charged spin- 0 , spin- $\frac{1}{2}$, Proca's spin-1 particles and to higher-spin particles phrased via 
the Bargmann-Wigner wave equation [15]. Generalization to external electromagnetic potential has been reported in Refs. [15, 19].

We will further argue that the above formulation can be looked at as if the particle would randomly propagate (in the sense of Brownian motion) through inhomogeneous or granular medium ("vacuum"). Here we will take this picture to its logical consequences, leaving the issue of actual dynamical or statistical origin of granularity of space for a future publication.

The Letter is organized as follows: in the next section, we settle the notation for superstatistics path integrals, explaining how the structure of smearing distributions and local Hamiltonians is generated via Lévy-Khinchine theorem. In Sect. 3 we display how the superstatistics paradigm can be used to analyse single-particle relativistic quantum dynamics in terms of two interlocked stochastic processes that operate at two separate spatio-temporal scales. Next we elucidate the physical meaning of our picture in the context of doubly-special relativity (DSR) where the DSR deformation is directly related with the small change in the mass-smearing distribution. The internal consistency of our proposal on the level of the second quantization is presented in Sect. 5 with the help of the worldline quantization. In Sect. 6 we present observational implications that our picture has in curved spacetimes. For specificity, we discuss Schwarzschild and Robertson-Walker spacetimes. In particular, be utilizing the Vilenkin-Ford model for inflationary cosmology we predict the upper bound of the leptogenesis period that is fitting within time window of a cosmologically constrained leptogenesis period. Finally in Sect. 7 we present our Conclusions and discuss further perspectives. In this Letter we do not discuss the technical issues related to reparametrization invariance. The interested reader can find the related bibliography, and proofs of the relevant statements and formulas in the accompanying paper [19].

\section{Superstatistics path integrals}

When a conditional probability density function (PDF) is formulated through a path integral (PI), then it satisfies the Einstein-Smoluchowski equation (ESE) for continuoustime Markovian processes, namely [20]

$p\left(x^{\prime}, t^{\prime} \mid x, t\right)=\int_{-\infty}^{\infty} \mathrm{d} y p\left(x^{\prime}, t^{\prime} \mid y, t^{\prime \prime}\right) p\left(y, t^{\prime \prime} \mid x, t\right)$,

with $t^{\prime \prime}$ being any time between $t^{\prime}$ and $t$. Conversely, any transition probability satisfying ESE possesses a PI representation [21]. In physics one often encounters conditional
PDFs formulated as a superposition of PIs, namely

$$
\begin{aligned}
& \wp\left(x^{\prime}, t^{\prime} \mid x, t\right) \\
& \quad=\int_{0}^{\infty} \mathrm{d} \zeta \omega(\zeta, T) \int_{x(t)=x}^{x\left(t^{\prime}\right)=x^{\prime}}[\mathrm{d} x][\mathrm{d} p] e^{\int_{t}^{t^{\prime}} \mathrm{d} \tau(\mathrm{i} p \dot{x}-\zeta H(p, x))} .
\end{aligned}
$$

Here $\omega(\zeta, T)$ with $T=t^{\prime}-t$ is a normalized PDF defined on $\mathbf{R}^{+} \times \mathbf{R}^{+}$. The form (3) typically appears in nonperturbative approximations to statistical partition functions, in polymer physics, in financial markets, in systems with reparametrization invariance, etc. [20]. The random variable $\zeta$ is then related to the inverse temperature, coupling constant, volatility, vielbein, etc.

The existence of different time scales and the flow of the information from slow to fast degrees of freedom typically creates the irreversibility on the macroscopic level of the description. The corresponding information thus is not lost, but passes in a form incompatible with the Markovian description. The most general class of distributions $\omega(\zeta, T)$ on $\mathbf{R}^{+} \times \mathbf{R}^{+}$for which the superposition of Markovian processes remain Markovian, i.e., when also $\wp\left(x^{\prime}, t^{\prime} \mid x, t\right)$ satisfies the ESE (2), was found in Ref. [14]. The key is to note that, in order to have (2) satisfied by $\wp$, the rescaled PDF $w(\zeta, T) \equiv \omega(\zeta / T, T) / T$ should satisfy the ESE for homogeneous Markov process

$w\left(\zeta, T_{1}+T_{2}\right)=\int_{0}^{\zeta} \mathrm{d} \zeta^{\prime} w\left(\zeta^{\prime}, T_{1}\right) w\left(\zeta-\zeta^{\prime}, T_{2}\right)$.

Consequently the Laplace image (moment-generating function) fulfills the functional equation

$\tilde{w}\left(p_{\zeta}, T_{1}+T_{2}\right)=\tilde{w}\left(p_{\zeta}, T_{2}\right) \tilde{w}\left(p_{\zeta}, T_{1}\right)$,

with $T_{1}, T_{2} \in \mathbf{R}^{+}$. By assuming continuity in $T$, it follows that the multiplicative semigroup $\tilde{w}\left(p_{\zeta}, T\right)_{T \geq 0}$ satisfies

$\tilde{w}\left(p_{\zeta}, T\right)=\left\{\tilde{w}\left(p_{\zeta}, 1\right)\right\}^{T}$.

So the distribution of $\zeta$ at $T$ is completely determined by the distribution of $\zeta$ at $T=1$. In addition, because $\tilde{w}\left(p_{\zeta}, 1\right)=$ $\left\{\tilde{w}\left(p_{\zeta}, 1 / n\right)\right\}^{n}$ for any $n \in \mathbf{N}^{+}, w(\zeta, 1)$ is infinitely divisible. The Lévy-Khinchine theorem $[17,18]$ then ensures that $\log \tilde{w}\left(p_{\zeta}, T\right) \equiv-T F\left(p_{\zeta}\right)$ must have the generic form

$\log \tilde{w}\left(p_{\zeta}, T\right)=-T\left(a p_{\zeta}+\int_{0}^{\infty}\left(1-e^{-p_{\zeta} u}\right) v(\mathrm{~d} u)\right)$,

where $a \geq 0$ is a drift constant and $v$ is some non-negative measure on $(0, \infty)$ satisfying $\int_{\mathbf{R}^{+}} \min (1, u) v(\mathrm{~d} u)<\infty$. Finally the Laplace inverse of $\tilde{w}\left(p_{\zeta}, T\right)$ yields $\omega(\zeta, T)$. Once 
$\omega(\zeta, T)$ is found, then $\wp\left(x^{\prime}, t^{\prime} \mid x, t\right)$ possesses a PI representation on its own. What is the form of the new Hamiltonian? To this end we rewrite (3) in the Dirac notation as

$$
\begin{aligned}
\wp\left(x^{\prime}, t^{\prime} \mid x, t\right) & =\left\langle x^{\prime}\left|\int_{0}^{\infty} \mathrm{d} \zeta w(\zeta, T) e^{-\zeta \hat{H}}\right| x\right\rangle \\
& =\left\langle x^{\prime}\left|\{\tilde{w}(\hat{H}, 1)\}^{T}\right| x^{\prime}\right\rangle=\left\langle x^{\prime}\left|e^{-T F(\hat{H})}\right| x^{\prime}\right\rangle .
\end{aligned}
$$

Hence, the identification $\mathscr{H}(p, x)=F(H(p, x))$ can be made. Here the Weyl ordering is implicitly assumed. In this case the mid-point rule follows automatically and one does not need to invoke gauge invariance to justify it $[14,20,22] .^{1}$

\section{Emergent special relativity}

The Feynman transition amplitudes (or better their Euclidean-version transition probabilities) naturally fits into the structure of superstatistics PIs discussed above. Note first that the choice $a=0$ and $v(\mathrm{~d} u)=1 /\left(2 \sqrt{\pi} u^{3 / 2}\right) \mathrm{d} u$ leads to $F\left(p_{\zeta}\right)=\sqrt{p_{\zeta}}$. This identifies $w(\zeta, T)$ with the (unshifted) Lévy distribution with the scale parameter $T^{2} / 2$. Moreover, when $H(\boldsymbol{p}, \boldsymbol{x})=\boldsymbol{p}^{2} c^{2}+m^{2} c^{4}$ then (3) can be cast into the form (see also Refs. $[14,15,19]$ )

$$
\begin{aligned}
\int_{\boldsymbol{x}(0)=x}^{\boldsymbol{x}(T)=\boldsymbol{x}^{\prime}}[\mathrm{d} \boldsymbol{x}][\mathrm{d} \boldsymbol{p}] \exp \left\{\int_{0}^{T} \mathrm{~d} \tau\left[\mathrm{i} \boldsymbol{p} \cdot \dot{\boldsymbol{x}}-c \sqrt{\boldsymbol{p}^{2}+m^{2} c^{2}}\right]\right\} \\
=\int_{0}^{\infty} \mathrm{d} \mu \phi_{\frac{1}{2}}\left(\mu, T c^{2}, T c^{2} m^{2}\right) \\
\quad \times \int_{\boldsymbol{x}(0)=\boldsymbol{x}}^{\boldsymbol{x}(T)=\boldsymbol{x}^{\prime}}[\mathrm{d} \boldsymbol{x}][\mathrm{d} \boldsymbol{p}] \exp \left\{\int_{0}^{T} \mathrm{~d} \tau[\mathrm{i} \boldsymbol{p} \cdot \dot{\boldsymbol{x}}\right. \\
\left.\left.\quad-\frac{\boldsymbol{p}^{2}}{2 \mu}-m c^{2}\right]\right\},
\end{aligned}
$$

where $T=t^{\prime}-t$, and

$\phi_{p}(z, a, b)=\frac{(a / b)^{p / 2}}{2 K_{p}(\sqrt{a b})} z^{p-1} \mathrm{e}^{-(a z+b / z) / 2}$,

is the generalized inverse Gaussian distribution [18] $\left(K_{p}\right.$ is the modified Bessel function of the second kind with index $p$ ). The left-hand side of (9) represents the PI for a free relativistic particle in the Newton-Wigner representation [23]. The genuine Klein-Gordon (KG) kernel which also contains negative-energy spectrum (reflecting the existence of the charge-conjugated solution-the antiparticle)

\footnotetext{
${ }^{1}$ Note different orderings may be required by certain symmetry principles [20]. For instance, while the mid-point prescription is consistent with ordinary gauge invariance and hence with the Weyl ordering, invariance under nonholonomic coordinate transformations yields different orderings $[20,51]$.
}

can be obtained from (9) by considering the FeshbachVillars representation of the $\mathrm{KG}$ equation and making the substitution [15]

$$
\begin{aligned}
& \phi_{\frac{1}{2}}\left(\mu, T c^{2}, T c^{2} m^{2}\right) \\
& \quad \mapsto \frac{1+\operatorname{sgn}(T) \sigma_{3}}{2} \phi_{\frac{1}{2}}\left(\mu,|T| c^{2},|T| c^{2} m^{2}\right) .
\end{aligned}
$$

The matrix nature of the smearing distribution $\left(\sigma_{3}\right.$ is the Pauli matrix) naturally includes the Feynman-Stuckelberg boundary condition and thus treats both particles and antiparticles in a symmetric way $[15,24]$. When the partition function is to be calculated, the trace will get rid of the $\operatorname{sgn}(T)$-term and $1 / 2$ in front of $\phi_{\frac{1}{2}}$ is turned to 1 .

The structure of (9) implies that the nuisance parameter $\mu$ can be interpreted as a Newtonian mass which takes on continuous values distributed according to $\phi_{\frac{1}{2}}$ given by (10) with $\langle\mu\rangle=m+1 / T c^{2}$ and $\operatorname{var}(\mu)=m / T c^{2}+2 / T^{2} c^{4}$. Fluctuations of the Newtonian mass can be then depicted as originating from particle's evolution in an inhomogeneous or granular ("polycrystalline") medium. Granularity, as known from solid-state systems, typically leads to corrections in the local dispersion relation [25] and hence to alterations in the local effective mass. The following picture thus emerges: on the short-distance scale, a non-relativistic particle can be envisaged as propagating via classical Brownian motion through a single grain with a local mass $\mu$. Because the fast-time-scale motion is Brownian, the local probability density matrix (PDM) conditioned on some fixed $\mu$ in a given grain is Gaussian

$\hat{\rho}(\boldsymbol{p}, T \mid \mu)=(T / \mu 2 \pi)^{3 / 2} \exp \left[-T \hat{\boldsymbol{p}}^{2} / 2 \mu\right]$.

This fast-time process has a time scale $\sim 1 / \mu c^{2}$. An averaged value of the local time scale represents a transient temporal scale $\left\langle 1 / \mu c^{2}\right\rangle=1 / m c^{2}$ which coincides with particle's Compton time $T_{C}$-i.e., the time for light to cross the particle's Compton wavelength. As the particle moves through a "grainy environment" the Newtonian mass $\mu$ fluctuates and the corresponding joint PDM will be $\hat{\rho}(\boldsymbol{p}, T ; \mu)=\phi_{\frac{1}{2}}\left(\mu, T c^{2}, T c^{2} m^{2}\right) \hat{\rho}(\boldsymbol{p}, T \mid \mu)$. At scales much longer than $T_{C}$ (long-distance scale) the marginal PDM describing the mass-averaged behavior is

$\hat{\rho}(\boldsymbol{p}, T)=\int_{0}^{\infty} \mathrm{d} \mu \phi_{\frac{1}{2}}\left(\mu, T c^{2}, T c^{2} m^{2}\right) \hat{\rho}(\boldsymbol{p}, T \mid \mu)$.

The matrix elements of $\hat{\rho}(\boldsymbol{p}, T)$ in the $\boldsymbol{x}$-basis are then clearly described by the PI (9). It should be stressed that above mass fluctuations have nothing to do with the Zitterbewegung which is caused by interference between positiveand negative-energy wave components, which in our derivation are decoupled. 
We may further observe that by coarse-graining the velocity over the time $T_{C}$ we have $\langle|\boldsymbol{v}|\rangle_{T_{C}}=\left.(\langle|\boldsymbol{p}|\rangle /\langle\mu\rangle)\right|_{T_{C}}=c$. So on a short-time scale of order $\lambda_{C}$ the KG particle propagates with an averaged velocity which is the speed of light $c$. But if one checks the particle's position at widely separated intervals $\left(\gg \lambda_{C}\right)$, then many directional reversals along a typical PI trajectory will take place, and the particle's net velocity will then be smaller than $c$, as it should be for a massive particle. The particle then acquires a sharp mass equal to Einstein's mass, and the process (not being hindered by fluctuating masses) is purely Brownian. This conclusion is in line with the Feynman checkerboard picture [19, 26, 27] to which it reduces in the case of $(1+1)$ D relativistic Dirac particle.

\section{Doubly special relativity}

Understanding the robustness of the emergent Special Relativity under small variations in the mass-smearing PDF can guide the study of the relation between Einsteinian SR and other deformed variants of SR, such as Magueijo-Smolin doubly SR or Amelino-Camelia's deformed SR [28-31] or (quantum) $\kappa$-Poincaré deformation of relativistic kinematics [32]. To actually realize this, one should explore the connection between $\delta \omega$ (or $\delta \phi_{1 / 2}$ ) and $\delta F$ which from (7) reads

$$
\begin{aligned}
e^{-T\left[F\left(p_{\zeta}\right)+\delta F\left(p_{\zeta}\right)\right]} \\
\quad=\int_{0}^{\infty} \mathrm{d} \zeta e^{-\zeta p_{\zeta}}[\omega(\zeta, T)+\delta \omega(\zeta, T)] .
\end{aligned}
$$

Requiring, for instance, that $\delta \omega(\zeta=0, T)=0$ and $\delta \zeta(\zeta=$ $0, T)=0$ (i.e., the new smearing PDF is again positively skewed with $\zeta \in \mathbf{R}^{+}$), and seeking $\delta \zeta$ in the form

$\delta \zeta(\zeta, T)=\zeta^{\alpha} \sum_{n=0}^{\infty} \varepsilon_{n}(T) \zeta^{n}, \quad \alpha \leq 1, \varepsilon_{n}(T) \ll 1$,

one arrives at $\delta F\left(p_{\zeta}\right)$ which admits Laurent expansion in powers of $\sqrt{p_{\zeta}}$. If we truncate the expansion after $\varepsilon_{1}$ (which corresponds to Pawula's truncation of the Kramers-Moyal expansion [14]) we obtain [19]

$\mathscr{H}(\boldsymbol{p})=\varepsilon_{1} / 4+\left(1+\varepsilon_{0} / 2\right) \sqrt{\boldsymbol{p}^{2} c^{2}+m^{2} c^{4}+\varepsilon_{2} / 4}$,

with $\varepsilon_{1}=-2\left(1+\varepsilon_{0} / 2\right) \sqrt{\varepsilon_{2}}$. This is particularly important in the context of doubly SR in which case (16) coincides with Magueijo-Smolin's Hamiltonian, in its version [33]. ${ }^{2}$

\footnotetext{
${ }^{2}$ It should be stressed that the Hamiltonian (16) (when also negative energy states are included) violates CPT. This is a typical byproduct of the Lorentz symmetry violation in many deformed SR systems.
}

\section{Quantum field theory}

The superstatistics transition probability (3) was constructed on the premise that $H$ (and ensuing $\mathscr{H}$ ) is associated with a single particle. Of course, the single-particle relativistic quantum theory is logically untenable, since a multiparticle production is allowed whenever a particle reaches the threshold energy for pair production. In addition, Leutwyler's no-interaction theorem [34, 35] prohibits interaction for any finite number of particles in the context of relativistic mechanics. To evade the no-interaction theorem it is necessary to have an infinite number of degrees of freedom to describe interaction. The latter is typically achieved via local quantum field theories (QFTs).

It should be underlined in this context that the PI for a single relativistic particle is still a perfectly legitimate building block even in QFT. Recall that in the standard perturbative treatment of, say, a generating functional for a scalar field each Feynman diagram is composed of integrals over product of free correlation functions (Feynman's correlators):

$\Delta_{F}\left(\boldsymbol{y}, c t_{y} ; \boldsymbol{z}, c t_{z}\right)=\frac{1}{4} \int_{-\infty}^{\infty} \mathrm{d} \tau \operatorname{sgn}\left(\tau-t_{y}\right) \wp\left(\boldsymbol{y}, \tau \mid z, t_{z}\right)$,

and may thus be considered as a functional of the path integral $\wp\left(\boldsymbol{x}^{\prime}, t^{\prime} \mid \boldsymbol{x}, t\right)$. In fact, QFT in general, can be viewed as a grand-canonical ensemble of fluctuating particle histories (worldlines) where Feynman diagrammatic representation of quantum fields depicts directly the pictures of the worldlines in a grand-canonical ensemble. In particular, the partition function for quantized relativistic fields can be fully rephrased in terms of single-particle relativistic PIs. This, the so-called "worldline quantization" of particle physics, is epitomized, e.g., in Feynman's worldline representation of the one-loop effective action in quantum electrodynamics [36, 37], in Kleinert's disorder field theory [38] or in the Bern-Kosower and Strassler "string-inspired" approaches to QFT [39-42]. For instance, the relationship between bosonic Bern-Kosower Green's function $G_{B}\left(\tau_{1}, \tau_{2}\right)$ and the transition probability $\wp\left(\boldsymbol{x}^{\prime}, \boldsymbol{t}^{\prime} \mid \boldsymbol{x}, t\right)$ can be found easily through the known functional relation between $G_{B}$ and $\Delta_{F}$, cf. Refs. [39-42].

\section{Gravity}

When the spacetime is curved, a metric tensor enters in both PIs in (9) in a different way, yielding different "counterterms" [20, 22]. In Bastianelli-van Nieuwenhuizen's time slicing regularization scheme [22] one, e.g., explicitly gets 
(when $\hbar$ is reintroduced) $)^{3}$

$$
\begin{aligned}
\frac{\boldsymbol{p}^{2}}{2 \tilde{m}} \mapsto \frac{g^{i j} p_{i} p_{j}}{2 \tilde{m}} & +\frac{\hbar^{2}}{8 \tilde{m}}\left(R+g^{i j} \Gamma_{i l}^{m} \Gamma_{j m}^{l}\right) \\
\sqrt{\boldsymbol{p}^{2}+m^{2} c^{2}} \mapsto & \sqrt{g^{i j} p_{i} p_{j}+\frac{\hbar^{2}}{4}\left(R+g^{i j} \Gamma_{i l}^{m} \Gamma_{j m}^{l}\right)+m^{2} c^{2}} \\
& +\hbar^{4} \Phi\left(R, \partial R, \partial^{2} R\right)+\mathscr{O}\left(\hbar^{6}\right) .
\end{aligned}
$$

Here $g^{i j}, R, \Gamma_{k l}^{j}$ and $\Phi$ are, respectively, the pull-back metric tensor, the scalar curvature, the Christoffel symbol, and a non-vanishing function of $R$ and its first two derivatives. This causes that the superstatistics identity (9) breaks down as can be explicitly checked to the lowest order in $\hbar$. The respective two cases will thus lead to different physics. Since the Einstein equivalence principle requires that the local spacetime structure can be identified with the Minkowski spacetime possessing Lorentz symmetry, one might assume the validity of (9) at least locally. The characteristic size of the local inertial (i.e. Minkowski) frame is of order $1 /|K|^{1 / 4}$ where $K=R_{\alpha \beta \gamma \delta} R^{\alpha \beta \gamma \delta}$ is the Kretschmann invariant and $R_{\alpha \beta \gamma \delta}$ is the Riemann curvature. So the breakdown of (9) happens when $\lambda_{C} \gtrsim 1 /|K|^{1 / 4}$. For instance, in the Schwarzschild geometry the breakdown should be expected at radial distances $r \lesssim\left(\lambda_{C}^{2} r_{s}\right)^{1 / 3}\left(r_{s}\right.$ is the Schwarzschild radius) which are-apart from the hypothetical case of micro-black holes (where $\lambda_{C} \simeq r_{s}$ ) —always deeply buried below the Schwarzschild event horizon. In the cosmologically relevant Robertson-Walker (RW) geometry the breakdown happens when $\left(\dot{a}^{4}+a^{2} \ddot{a}^{2}\right) \gtrsim\left(a c / \lambda_{C}\right)^{4}$, where $a(t)$ is the RW scale factor of the Universe and $\dot{a}=$ $\mathrm{d} a / \mathrm{d} t$. Applying the well-known Vilenkin-Ford model [43, 44] for inflationary cosmology, where $a(t)=A \sqrt{\sinh (B t)}$ with $B=2 c \sqrt{\Lambda / 3}$ ( $\Lambda$ is the cosmological constant), we obtain

$t \lesssim \frac{1}{B} \operatorname{arcsinh}\left[\frac{B \lambda_{C}}{\left(8 c^{4}-\left(B \lambda_{C}\right)^{4}\right)^{1 / 4}}\right] \equiv \bar{t}$.

By using the presently known [45] value $\Lambda \simeq 10^{-52} \mathrm{~m}^{-2}$ and the $\tau$-lepton Compton's wavelength $\lambda_{C}^{\tau} \simeq 6.7 \times 10^{-16} \mathrm{~m}$ (yielding the tightest upper bound on $t$ ), we obtain $\bar{t} \simeq$ $4 \times 10^{-24} \mathrm{~s}$. Such a violation of the local Lorentz invariance naturally breaks the particle-antiparticle symmetry since there is no unified theory of particles and antiparticles in the non-relativistic physics-formally one has two separate theories. The ensuing matter-antimatter asymmetry might be relevant in the early Universe, e.g., for leptogenesis. In

\footnotetext{
${ }^{3}$ Analogous disparity behavior between both PIs can be directly obtained also from Cherviakov-Kleinert curved-space quantization based on the non-holonomic mapping principle $[20,51]$.
}

this respect, $\bar{t}$ is consistent with the nonthermal leptogenesis period that typically dates between $10^{-26}-10^{-12} \mathrm{~s}$ after the big bang.

\section{Conclusions and perspectives}

The superstatistics PI representation of a relativistic point particle presented in this Letter, realizes an explicit quantum mechanical duality between Einsteinian and Galilean relativity. It also makes explicit how the SR invariance is encoded in the grain smearing distribution. Notably, the exact Lorentz symmetry of a spacetime has no fundamental significance in our analysis, as it is only an accidental symmetry of the coarse-grained configuration space in which a particle executes a standard Wiener process. In the passage from grain to grain, particle's Newtonian mass fluctuates according to an inverse Gaussian PDF. This might be viewed as a refinement of the world crystal model proposed in Ref. [46].

The presented PI representation when embedded in the QFT framework may help to study several cosmological implications of systems with granular space. If any of such systems quickly flows to the infrared fixed point, any direct effect from the space discreteness and related SR violation might be insignificant on cosmological scales (where Lorentz and diffeomorphism invariance are restored), while it might be crucial in the early Universe, e.g., for leptogenesis and the ensuing baryogenesis. Consequences on the detailed structure of the Cosmic Microwave Background Spectrum are certainly worth of being explored in future studies.

Finally, our model should reinforce the links between the superstatistics paradigm and the approach to quantum gravity based on stochastic quantization [47-50]. In particular, the outlined granular space could be a natural model for the noise terms in a Parisi-Wu stochastic-like quantization of gravity.

Acknowledgements We are grateful to Z. Haba, M. Sakellariadou, L.S. Schulman and H. Kleinert for useful feedback. This work was supported by GAČR Grant No. P402/12/J077.

Open Access This article is distributed under the terms of the Creative Commons Attribution License which permits any use, distribution, and reproduction in any medium, provided the original author(s) and the source are credited.

\section{References}

1. P. Anderson, Science 177, 393 (1972)

2. R.B. Laughlin, A Different Universe: Reinventing Physics from the Bottom Down (Basic Books, London, 2005)

3. I. Licata, A. Sakaji, Physics of Emergence and Organization (World Scientific, London, 2008)

4. C. Beck, Phys. Rev. Lett. 87, 180601 (2001)

5. C. Beck, E.G.D. Cohen, Physica A 322, 267 (2003) 
6. G. Wilk, Z. Wlodarczyk, Phys. Rev. Lett. 84, 2770 (2000)

7. R. Hanel, S. Thurner, M. Gell-Mann, Proc. Natl. Acad. Sci. USA 108, 6390 (2011)

8. A. Reynolds, Phys. Rev. Lett. 91, 084503 (2003)

9. C. Beck, S. Miah, arXiv:1207.4062 (2012)

10. K.E. Daniels, C. Beck, E. Bodenschatz, Physica D 193, 208 (2004)

11. G. Wilk, Z. Wlodarczyk, Eur. Phys. J. A 40, 299 (2009)

12. C. Beck, Eur. Phys. J. A 40, 267 (2009)

13. C. Beck, in Anomalous Transport: Foundations and Applications, ed. by G. Radons et al. (Wiley, London, 2007)

14. P. Jizba, H. Kleinert, Phys. Rev. E 78, 031122 (2008)

15. P. Jizba, H. Kleinert, Phys. Rev. D 82, 085016 (2010)

16. P. Allegrini, F. Barbi, P. Grigolini, P. Paradisi, Phys. Rev. E 73, 046136 (2006)

17. J. Bertoin, Lévy Processes (Cambridge University Press, Cambridge, 1996)

18. W. Feller, An Introduction to Probability Theory and Its Applications, vol. II (Wiley, London, 1966)

19. P. Jizba, F. Scardigli, Phys. Rev. D 86, 025029 (2012)

20. H. Kleinert, Path Integrals in Quantum Mechanics, Statistics, Polymer Physics and Financial Markets, 4th edn. (World Scientific, Singapore, 2009)

21. R.P. Feynman, A.R. Hibbs, Quantum Mechanics and Path Integrals (McGraw-Hill, New York, 1965)

22. F. Bastianelli, P. van Nieuwenhuizen, Path Integrals and Anomalies in Curved Space (Cambridge University Press, Cambridge, 2005)

23. T. Newton, E. Wigner, Rev. Mod. Phys. 21, 400 (1949)

24. H. Feshbach, F. Villars, Rev. Mod. Phys. 30, 24 (1958)

25. E.A. Johnson, A. MacKinnon, J. Phys. Condens. Matter 5, 5859 (1993)

26. B. Gaveau, T. Jacobson, M. Kac, L.S. Schulman, Phys. Rev. Lett. 53, 419 (1984)
27. T. Jacobson, L.S. Schulman, J. Phys. A 17, 375 (1984)

28. G. Amelino-Camelia, Int. J. Mod. Phys. D 11, 35 (2002)

29. J. Magueijo, L. Smolin, Phys. Rev. Lett. 88, 190403 (2002)

30. G. Amelino-Camelia, Nature 418, 34 (2002)

31. J. Kowalski-Glikman, Introduction to Doubly Special Relativity. Lecture Notes in Physics, vol. 669 (Springer, Berlin, 2005)

32. M. Daszkiewicz, J. Lukierski, M. Woronowicz, Phys. Rev. D 77, 105007 (2008)

33. J. Magueijo, L. Smolin, Phys. Rev. D 67, 044017 (2003)

34. H. Leutwyler, Nuovo Cimento 37, 556 (1965)

35. G. Marmo, N. Mukunda, E.C.G. Sudarshan, Phys. Rev. D 30, 2110 (1984)

36. R.P. Feynman, Phys. Rev. 80, 440 (1950)

37. R.P. Feynman, Phys. Rev. 84, 108 (1951)

38. H. Kleinert, Gauge Fields in Condensed Matter, Vol. I. Superflow and Vortex Lines (World Scientific, Singapore, 1989)

39. Z. Bern, D.A. Kosower, Phys. Rev. Lett. 66, 1669 (1991)

40. Z. Bern, D.A. Kosower, Nucl. Phys. B 379, 451 (1992)

41. M.J. Strassler, Nucl. Phys. B 385, 145 (1992)

42. C. Schubert, Phys. Rep. 335, 73 (2001)

43. A. Vilenkin, L.H. Ford, Phys. Rev. D 26, 1231 (1982)

44. F. Scardigli, C. Gruber, P. Chen, Phys. Rev. D 83, 063507 (2011)

45. M. Tegmark et al., Phys. Rev. D 74, 123507 (2006)

46. H. Kleinert, Multivalued Fields in Condensed Matter, Electromagnetism, and Gravitation (World Scientific, Singapore, 2008)

47. H. Rumpf, Phys. Rev. D 33, 185 (1986)

48. H. Rumpf, Prog. Theor. Phys. Suppl. 111, 63 (1993)

49. T.C. de Aguiar, G. Menezes, N.F. Svaiter, Class. Quantum Gravity 26, 075003 (2009)

50. J. Ambjorn, R. Loll, W. Westra, S. Zohren, Phys. Lett. B 680, 359 (2009)

51. H. Kleinert, Gen. Relativ. Gravit. 32, 769 (2000) 